\title{
Foot Pressure Distribution Variation in Pre-obese, Obese and Non-obese Individuals - Forensic Implications
}

\author{
Kewal Krishan ${ }^{1, *}$ and Tanuj Kanchan ${ }^{2}$ \\ ${ }^{I}$ Department of Anthropology, Panjab University, Chandigarh, India \\ ${ }^{2}$ Department of Forensic Medicine, Kasturba Medical College, Mangalore, Manipal University, India
}

\begin{abstract}
The present commentary refers to recent research on the plantar pressure distribution variation in pre-obese, obese and non-obese adult individuals. While the studies observe significant changes in the contact area in the mid foot region i.e. instep region, no statistically significant differences were observed in the pressure distribution of the fore-foot and hind-foot region among the pre-obese and non-obese groups. Thus, the pressure distribution of the sole of the individuals depends upon the body weight of the individuals. In this commentary, we emphasize that the results of these studies; besides their clinical implications, have applications in forensic sciences especially in the field of forensic podiatry too which is concerned with the examination of pedal evidence recovered at the crime scene.
\end{abstract}

Keywords: Plantar pressure distribution, Forensic podiatry, Personal identification.

\section{COMMENTARY}

Plantar pressure distribution is likely to provide useful information in clinical as well as forensic practice. This commentary refers a recent research on the plantar pressure distribution in pre-obese, obese and non-obese adult individuals [1-4]. While these studies observe significant changes in the contact area in the mid foot region i.e. instep region, no statistically significant differences were observed in the pressure distribution of the fore-foot and hind-foot region among the pre-obese and non-obese groups. In a study conducted by Cousins et al. [5], the foot patterns show significantly greater loading at the midfoot and 2nd-5th metatarsals in children. Arnold et al. [6] also conducted a similar study where impact of increasing body mass has been investigated under four different loading conditions i.e. 0, 5, 10 , and $15 \mathrm{~kg}$. They observed statistically significant increases in peak pressure between the 10 and $15 \mathrm{~kg}$ load conditions compared to the control $(0 \mathrm{~kg})$ within the heel and second to fifth metatarsal regions. It can be well interpreted from the above mentioned studies that the obese individuals showed flatter foot pattern, larger foot axis angle and differences on dynamic plantar pressures compared to the non-obese individuals (Fig. 1). In other words, in case of pre-obese and obese individuals, the instep region is more impressed than those of the non-obese individuals. Thus, the pressure distribution of the sole of the individuals depends upon the body weight of the individuals.

The results of these studies; besides their clinical implications, have applications in forensic sciences

*Address correspondence to this author at the Department of Anthropology, Panjab University, Sector-14, Chandigarh, India; Tel: +919876048205;

E-mail: gargkk@yahoo.com especially in the field of forensic podiatry which deals with the examination of pedal evidence recovered at the crime scene. Various kinds of bare foot impressions are often recovered at the crime scenes. These impressions are inadvertently left in the blood, water, mud, and freshly made floors etc. The results of the study can be of significance in analysis of footprints for predicting the body size of the perpetrator. In this regard, the effect of additional weight on the footprints has been studied by researchers [7]. The dimensions of the footprints were significantly affected when the individuals were given additional weight of 20 kilograms. Significant changes in the contact area in mid foot regions between the pre-obese and obese groups as observed in the studies on plantar pressure distribution can give a clue to the body size from a footprint.

At times, footwear may be recovered from the crime scenes. The foot impressions on the insoles of the footwear are also studied during criminal investigations. These impressions available on the insoles of the footwear can be matched with the footprints available at the crime scenes. These patterns found on the insoles of the footwear are also individualistic to a person who wore this footwear [8]. The findings of research on plantar pressure distribution may be helpful in understanding the pattern on the insole of the footwear, and also supportive in interpreting the body size of the holder of the footwear.

The observations of the published literature on plantar pressure distribution pertain to static footprints since these studies were conducted on standing individuals. Since dynamic footprints are encountered frequently in real forensic situations, further studies on dynamic footprints are proposed. We propose that in future researches, foot pressure distribution of the non-obese, pre-obese and obese individuals can be compared and the results be interpreted in 




\section{$\begin{array}{ll}\text { (A) An obese individual } & \text { (B) A non-obese individual }\end{array}$}

Fig. (1). Footprints showing impression of the plantar surface of the obese and non-obese individuals.

forensic terms. These studies would be helpful in predicting the body size of the individuals from the footprints, which may prove to be helpful during the criminal investigations.

\section{CONFLICT OF INTEREST}

The author confirms that this article content has no conflict of interest.

\section{ACKNOWLEDGEMENTS}

This commentary has emerged from the results of a major research project entitled "Association of foot with footwear-A forensic study based on characteristic impressions of soles on insoles of rubber slippers" awarded by University Grants Commission, New Delhi, India to Dr Kewal Krishan vide grant F.No. 34-120/2008 (SR) dated $2^{\text {nd }}$ January 2009.

\section{REFERENCES}

Periyasamy R, Mishra A, Anand S, Ammini AC. Foot pressure distribution variation in pre-obese and non-obese adult subject while standing. Foot (Edinb) 2012; 22: 276-82.
[2] Song-hua Y, Zhang K, Tan GQ, Yang J, Liu ZC. Effects of obesity on dynamic plantar pressure distribution in Chinese prepubescent children during walking. Gait Posture 2013; 37: 37-42.

[3] Periyasamy R, Anand S, Ammini AC. Prevalence of standing plantar pressure distribution variation in north Asian Indian patients with diabetes mellitus: a study to understand ulcer formation. Proc Inst Mech Eng H J Eng Med 2013; 227: 181-9.

[4] Joob B, Wiwanitkit V. Foot pressure distribution variation in preobese and non-obese adult. Foot (Edinb) 2013; 23: 53.

[5] Cousins SD, Morrison SC, Drechsler WI. Foot loading patterns in normal weight overweight and obese children aged 7 to 11 years. J Foot Ankle Res 2013; 6(1): 36.

[6] Arnold JB, Causby R, Pod GD, Jones S. The impact of increasing body mass on peak and mean plantar pressure in asymptomatic adult subjects during walking. Diabet Foot Ankle 2010; 1. doi: 10.3402/dfa.v1i0.5518.

[7] Krishan K. Establishing correlation of footprints with body weightforensic aspects. Forensic Sci Int 2008; 179: 63-9.

[8] DiMaggio JA, Vernon W. Forensic Podiatry-Principles and Methods. Springer: New York, Dordrecht Heidelberg, London: Humana Press. 2011.

(C) Krishan and Kanchan; Licensee Bentham Open.

This is an open access article licensed under the terms of the Creative Commons Attribution Non-Commercial License (http://creativecommons.org/licenses/by-nc/3.0/) which permits unrestricted, non-commercial use, distribution and reproduction in any medium, provided the work is properly cited. 